\title{
Knowledge Dynamics and Innovation: Case Studies in a Sparsely Populated Area
}

\begin{abstract}
:
This study describes the sources of essential knowledge for the innovation cases in four micro-sized companies and analyses the knowledge dynamics in these cases. This study seeks to answer the following questions: What are the sources of essential knowledge for the cases selected? What kind of knowledge is required, and what phases can be identified? What kind of features of knowledge anchoring can be identified in the innovation processes? These questions are answered through a multiple case study with the knowledge biography technique. This study will serve as an interesting benchmarking tool for public organisations that offer business services to companies. The findings suggest that public actors should pay more attention to the case-specific needs of innovative micro-sized companies while developing their services. In the future studies, the understanding of knowledge dynamics could be deepened by researching learning mechanisms and knowledge management practices that influence the innovations of micro-sized companies.
\end{abstract}

Keywords: knowledge dynamics, knowledge biography, knowledge anchoring, innovation, learning, micro-sized company, growth, Finland

\section{Introduction}

Knowledge is generally connected to innovation. In fact, changes in knowledge can be seen as the driving force behind innovation, and the general product/innovation life cycle can be divided into generic phases, stages or states in multiple ways (e.g. Dean, 1950; Golder and Tellis, 2004; Markusen et al., 1986; Schutjens and Stam, 2003). This study aims to describe the sources of knowledge that are essential for the innovation cases in four micro-sized companies located in Oulu South region and analyse the knowledge dynamics in these cases.

The Oulu South region is situated in the southern part of Northern Ostrobothnia in Finland. This region is not a governmental unit or area but was formed to increase intermunicipal cooperation and development and to gain the required critical mass to ensure national and international competitiveness. The region comprises three subregions and 14 municipalities, with approximately 90,000 inhabitants and 4,600 active companies. Oulu South is one of Finland's main rural areas; however, because it offers sufficient employment to its inhabitants, it is known as the 'industrialised countryside'. Furthermore, although the unemployment rate in this region is the lowest in northern Finland, it is likely to retain a large proportion of its younger population because it has the highest birth rate in Finland, despite the demographic challenge of widespread emigration from the region. This distinguishes the Oulu South region from other rural areas in Finland, many of which are dealing with significantly ageing populations.

In this study, we investigate four innovation cases in micro-sized companies located in the Oulu South region:

Case 1. Development of a vertical lifting fabric fold-up outer door. 
Case 2. Development of a method to process and convert the ash generated by power plants to end-of-waste products.

Case 3. Development of an Internet application to describe electricity price formation and contract rating.

Case 4. Development of a PC-free control system for a forest harvester with remote control possibilities.

This study focuses on the knowledge dynamics of these four innovation cases to garner a better understanding of how knowledge is developed and transferred at a firm level. The aim of studying knowledge dynamics is to unravel the processes of knowledge interactions and to identify the types of actors that are involved in these processes. Therefore, we defined the following research questions:

1. What are the sources of knowledge that are essential for the four innovation cases?

2. What kind of knowledge is required and what phases can be identified in the four innovation cases?

3. What kind of features of knowledge anchoring can be identified in the four innovation processes?

To address these research questions, multiple case studies were conducted. This was deemed the optimum approach for the purposes of our study. According to Yin (1989, p. $23)$, 'a case study is an empirical inquiry that investigates a contemporary phenomenon within its real-life context, when the boundaries between phenomenon and context are not evident and in which multiple sources of evidence are used'. An essential part of this study was to conduct in-depth interviews with the key informants of each innovation case. Because this study deals with interactions among actors and particularly aims to evaluate knowledge exchange and development, this was deemed the best way to obtain considerable information regarding these processes. The information obtained through the interviews was analysed predominantly by using the knowledge biography technique.

This study may enable public actors to ask essential questions for developing their services for micro-sized companies, thereby fostering regional development and economic growth. According to Crevoisier and Jeannerat (2009, p. 1228), those regions that are capable of imagining their local activities within a global environment are categorised as innovative regions. To be innovative, a region must be capable of matching its dynamics to the generation and utilisation of knowledge.

This study is made up of five sections. In the introduction, the background, motivation, research problem and research questions are presented. In the second section, the theoretical background of the study is presented and a brief survey of the relevant knowledge dynamics, types, phases and anchoring is conducted. In the third section, the method undertaken in this study is described. In the fourth section, description and analysis of the data garnered in the case studies are undertaken; these findings are then examined in light of the research questions. In the final section, a discussion of the main results and opportunities for further research is presented.

\section{Knowledge Dynamics}

Knowledge is widely regarded as a useful economic resource. In this context, it has been defined as the learning process in human brains, which is generated and used in personal and collective interactions in various contexts and uses given individual and firm 
competences to appropriate new and necessary economically useful knowledge (Dahlström and Hedin, 2010, p. 6). Therefore, knowledge is seen as both a resource and a process, both of which are linked to the interactions that occur among actors in the concept of knowledge dynamics. According to Strambach (2008, p. 154), the visible results of knowledge dynamics are innovations in products, services or processes. Knowledge dynamics can be understood as the dynamics that emerge from the processes of creation, usage, transformation, movement and diffusion of knowledge. Knowledge is not static; rather, it is constantly changing and dependent on context and application.

\subsection{Knowledge types, knowledge phases and knowledge process}

For the purposes of this empirical case study, analytical, synthetic and symbolic knowledge were considered most relevant. Analytical knowledge is defined as researchbased knowledge, which is developed through scientific exploration. Synthetic knowledge is considered a combination of analytical and (perhaps) symbolic knowledge. Engineering knowledge is a good example of synthetic knowledge, because it is derived from application and scientific research. Symbolic knowledge is largely recognised in research on cultural or creative industries. It deals with ideas, symbols and socially constructed commodities. It has been referred to as the knowledge about representation (Asheim and Coenen, 2006; Strambach, 2008).

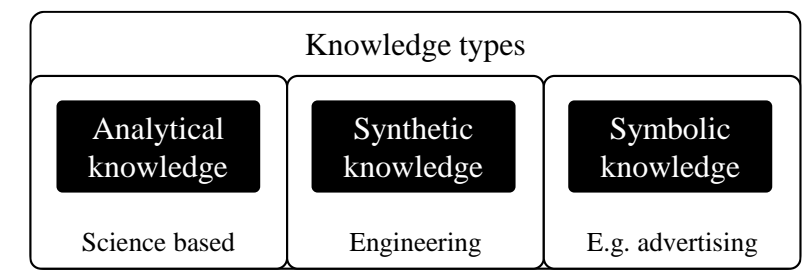

Figure 1. Types of knowledge

A distinction is often made between tacit and codified knowledge when examining knowledge types. Codified knowledge can be transmitted relatively easily to others because it is understood to be knowledge that can be represented either in writing or in digital or analogue format. In contrast, tacit knowledge is understood to be knowledge that is cultivated largely through practice and is embodied in people. It is articulated through practical skills and cannot be reduced to numbers, graphs, maps, diagrams or texts, etc. Face-to-face contact or 'buzz' is, consequently, an important part of tacit knowledge transfer (Collinge et al., 2008; Halkier et al., 2010).

Knowledge phases seldom progress linearly. Usually, development progresses in such a way that various phases occur at the same time. In fact, there may even be loops between these phases. To understand the complexity of knowledge dynamics as a part of the process of innovation, the following three knowledge phases are examined in this study: exploration, examination and exploitation. 


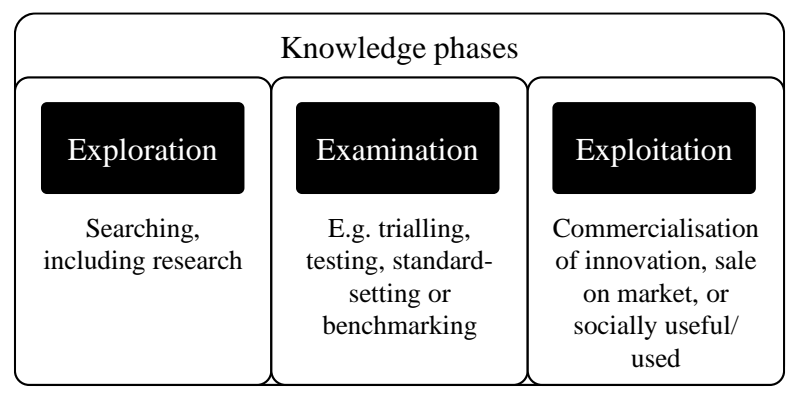

Figure 2. Knowledge phases

The exploration phase is often described as the first step in the knowledge chain. This phase usually involves searching for new knowledge or maintaining and developing existing knowledge. Examination is a testing phase in which the veracity and applicability of the knowledge is considered. Exploitation is regarded as a 'selling' and 'using' phase, in which knowledge is applied to a situation in return for financial, status, position or recognition gains (Halkier et al., 2010; Strambach, 2008).

\subsection{Knowledge anchoring}

The importance of innovation and knowledge transfer in the regional economic development process has increasingly been emphasised. The concept of knowledge anchoring refers to the ability of an organisation or territory to access external knowledge and make use of it in some way. Anussornnitisarn et al. (2010), Kess et al. (2008) and Phusavat et al. (2009) have highlighted the importance of external knowledge for organisational learning.

James et al. (2010, p. 60) define knowledge anchoring as that knowledge which originates outside of a particular region, but that, in some way, 'sinks in' and is recirculated within that region. It also refers to the processes through which knowledge is used by other firms/institutions within a region (not just the one that found/adopted the knowledge from an external source). This might include developing new knowledge, recombining this external knowledge with existing knowledge and diffusing the knowledge within the region.

The concept of knowledge anchoring is a useful tool with which one can analyse the different mechanisms through which knowledge flows into, and is recirculated within, regions and firms. In this study, the knowledge anchoring is viewed in the firm-level context. The inflow and recirculation of knowledge may occur at the same time and in complex mixes of processes. Knowledge anchoring through firm-level interaction refers, for instance, to interaction through organised networks and direct interactions between companies as well as to unmediated and mediated interactions. This functions only insofar as the individuals that interact do so as representatives of firms, organisations and knowledge institutions. Examples of activities relevant to workplace or job-related mobility are people moving to a region, movements of employees within an organisation and business trips. Acquisition of codified knowledge is often connected with academic activities such as events or publications. Gaining access to this can be undertaken through, for example, browsing the Internet and reading publications. Events are arenas for recirculation of knowledge, in which expected and unexpected knowledge interactions can occur because people who would not otherwise meet are brought together, either inside or outside the region (Dahlström and Hedin, 2010, p.6-7, 82-93). 
Knowledge anchoring is useful for analysing the important aspects of knowledge dynamics. It includes analysing characteristics of knowledge interactions. Knowledge interactions are characterised by their cross-sectoral, multiscalar and multiactor nature. Cross-sectoral knowledge interactions are innovative and drive product development. Territorial and firm-level knowledge dynamics include extraregional interactions. Multiactor nature means that there are many types of actors, both private and public, in knowledge interactions. By understanding knowledge anchoring processes better, policies on knowledge interactions, innovations and growth can be selected for adoption that match regional needs as closely as possible. (Dahlström and Hedin, 2010, p. 126-127)

\section{The Method}

We used a multiple case study with holistic strategy. The case study followed the process presented in Figure 3.

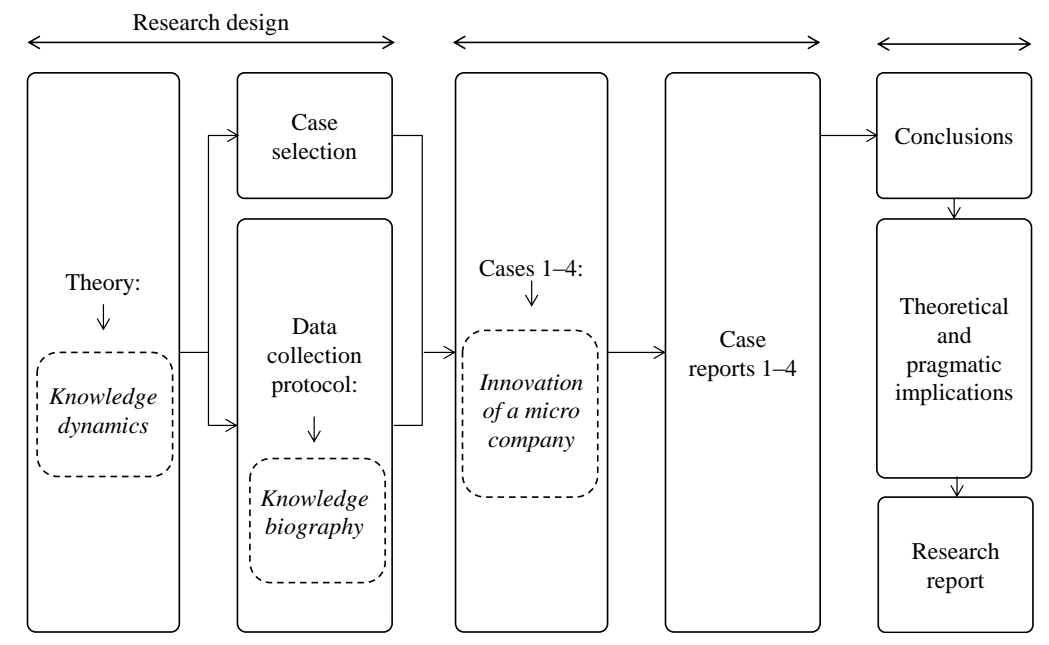

Figure 3. The research process

The study analysed the process of innovation cases in four micro-sized companies. The definition of a micro-sized company (and small- and medium-sized enterprise, SME) is different in different contexts. The US context, for example, offers many definitions of SME, depending on the industry. For example, in some industries, an SME is defined as any company having fewer than 500 employees. In the European context, an SME is defined as any company having fewer than 250 employees. The same disparity applies to micro-sized companies and should be taken into account when studying the SME-related literature. Ayyagari et al. (2007) cover this topic in their globally focused statistical study on SMEs (p. 416): 'The term SME covers a wide range of definitions and measures, varying from country to country and varying between sources reporting SME statistics. Some of the commonly used criteria are the number of employees, total net assets, sales, and investment level. However, the most common basis for definition is employment, and here again, there is variation in defining the upper and lower size limit of an SME. Despite this variance, a large number of sources define an SME to have a cut-off of 250 employees'. Because of the European context of this case study, we utilise the European definition. Within the SME category, the European Union defines medium-sized firms as 
having 50-249 employees, small firms as having 10-49 employees and micro-sized firms as having 0-9 employees (Storey, 2003).

In this study, the empirical data was gathered through semi-structured interviews and public archives related to each company in question. Most of the interviews with key informants were conducted face-to-face during fieldwork. The questionnaires were designed to obtain information from the informants on various questions including the following: How did the innovation process develop from idea to product? What important knowledge interactions occurred during the development of the product? Who were the main actors involved in the development process? Where were they located? What type of knowledge did they contribute to the process? What core knowledge was involved in the genesis and development of this innovation? How did the core knowledge of the innovation arise and how was it developed? During each interview, the interviewer summarised the explanations provided by the interviewees in order to avoid a biased or incomplete interpretation. Each case study evidence was then saved in the database. Each case study evidence was collected by a researcher trained in data collection procedures and familiar with the theoretical background to this study. The data collection process took place in 2013 (cases 1-3) and 2008-2009 (case 4); following this, an inclusive and iterative process was used to analyse the data, and the main findings were summarised.

The knowledge biography technique - an innovative approach that provides a deeper understanding of knowledge dynamics in firms and regions-was used as the main technique for interpreting and analysing the data. The essential sources of knowledge for each innovation case were tracked, and interviews were used to obtain basic information regarding the time and space dimensions of the knowledge dynamics that occurred as a part of the cases. The knowledge biography technique permitted us to capture the diversity of the social environment of each firm to determine the precise manner in which knowledge was transmitted, and information was exchanged from various partners inside and outside the firm (Dahlström and Hedin, 2010, p. 11).

\section{Case Studies}

In this section, we describe the essential knowledge sources for the four innovation cases and analyse the knowledge dynamics in these cases. Moreover, the detailed knowledge biography of each innovation case is presented.

The four innovation cases analysed in this study are as follows:

Case 1. Development of a vertical lifting fabric fold-up outer door.

Case 2. Development of a method to process and convert the ash generated by power plants to end-of-waste products.

Case 3. Development of an Internet application to describe electricity price formation and contract rating.

Case 4. Development of a PC-free control system for a forest harvester with remote control possibilities.

\subsection{Case 1}

Innovation case 1 is the development of a vertical lifting fabric fold-up outer door. Based on the data obtained from the interviews, three innovation phases were identified:

1. First version, 1993-1998

2. Product development, 1999-2004 
3. Marketing and internationalisation, 2005-

Figure 4 illustrates the innovation phases and key knowledge interactions among the actors in case 1 . The owner of the innovation remained the same (i.e., company 1) during the development process.

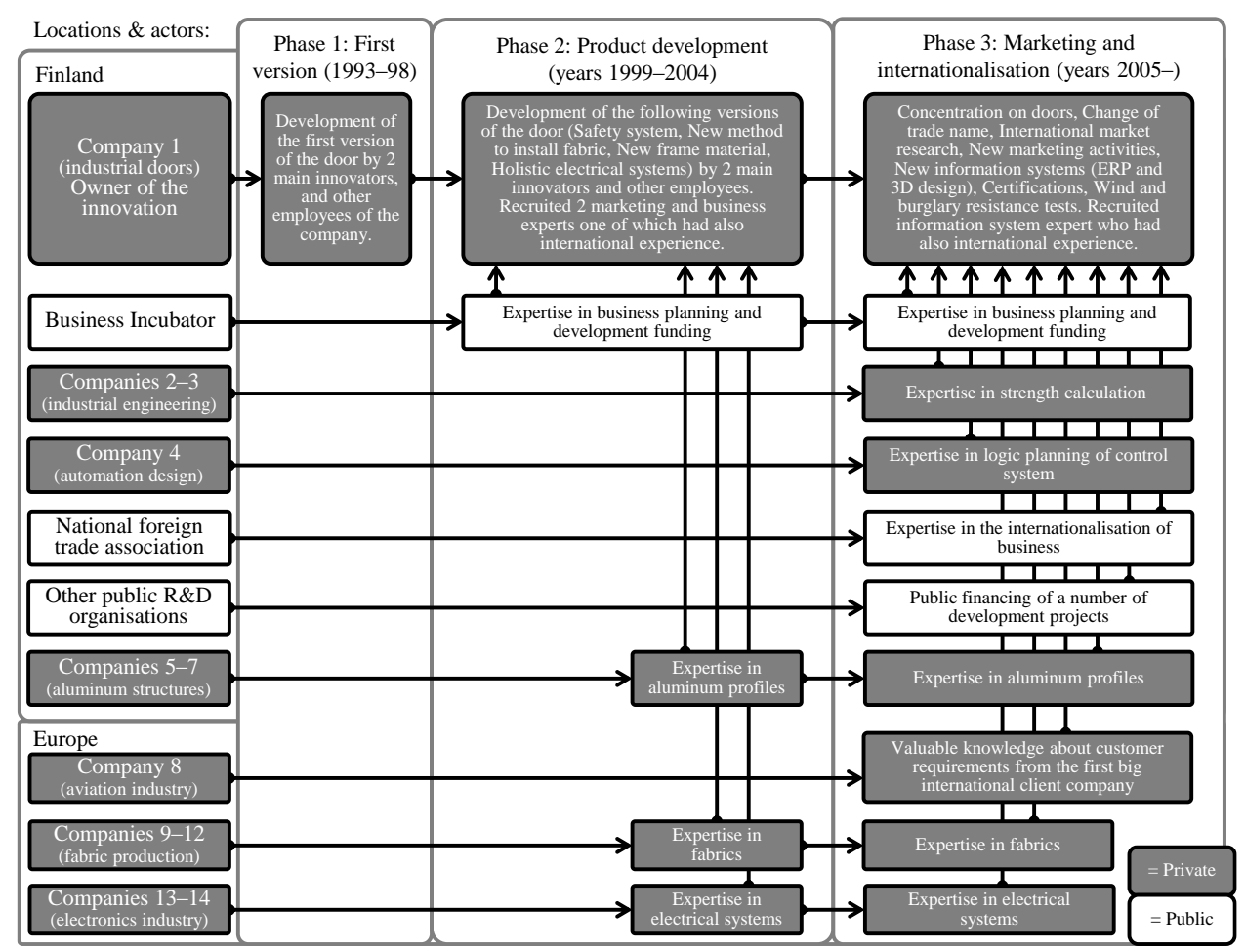

Figure 4. Knowledge biography of innovation case 1

The idea was based on the following question posed by one customer in 1993: 'Could you make us an outer door with a basic structure that is similar to your indoor divider wall?' Two main innovators made a plan for modifying the divider wall to outer door. Subsequently, the first version of an outer door was manufactured and sold to the customer in 1993. The entire planning and manufacturing process was completed by the staff of company 1 in phase 1 .

The employees of company 1 , who had considerable experience in manufacturing tailor-made sunblinds, sunshades and divider walls, contributed the most important knowledge to the development process. In phase 2, the expertise of several external experts was introduced to the process, mainly by the supplier companies. In phase 3 , internationalisation expertise was introduced to the development process by a public organisation, and knowledge regarding customer requirements was introduced to the process by the first big international client company. The supplier companies also introduced important external expertise to the process in phase 3 . 


\subsection{Case 2}

Innovation case 2 is the development of a processing method for ash from power plants. Based on the data obtained from the interviews, three innovation phases were identified:

1. Ideation, 2002-2008

2. Product development and marketing, 2009-2011

3. Commercialisation, 2012-

Figure 5 illustrates the innovation phases and key knowledge interactions among the actors in case 2. Two individuals (i.e., the main innovators) were the owners of the innovation in phase 1 . Subsequently, the company (i.e., company 1 ), which was founded in 2009 by the main innovators, was the owner of the innovation.

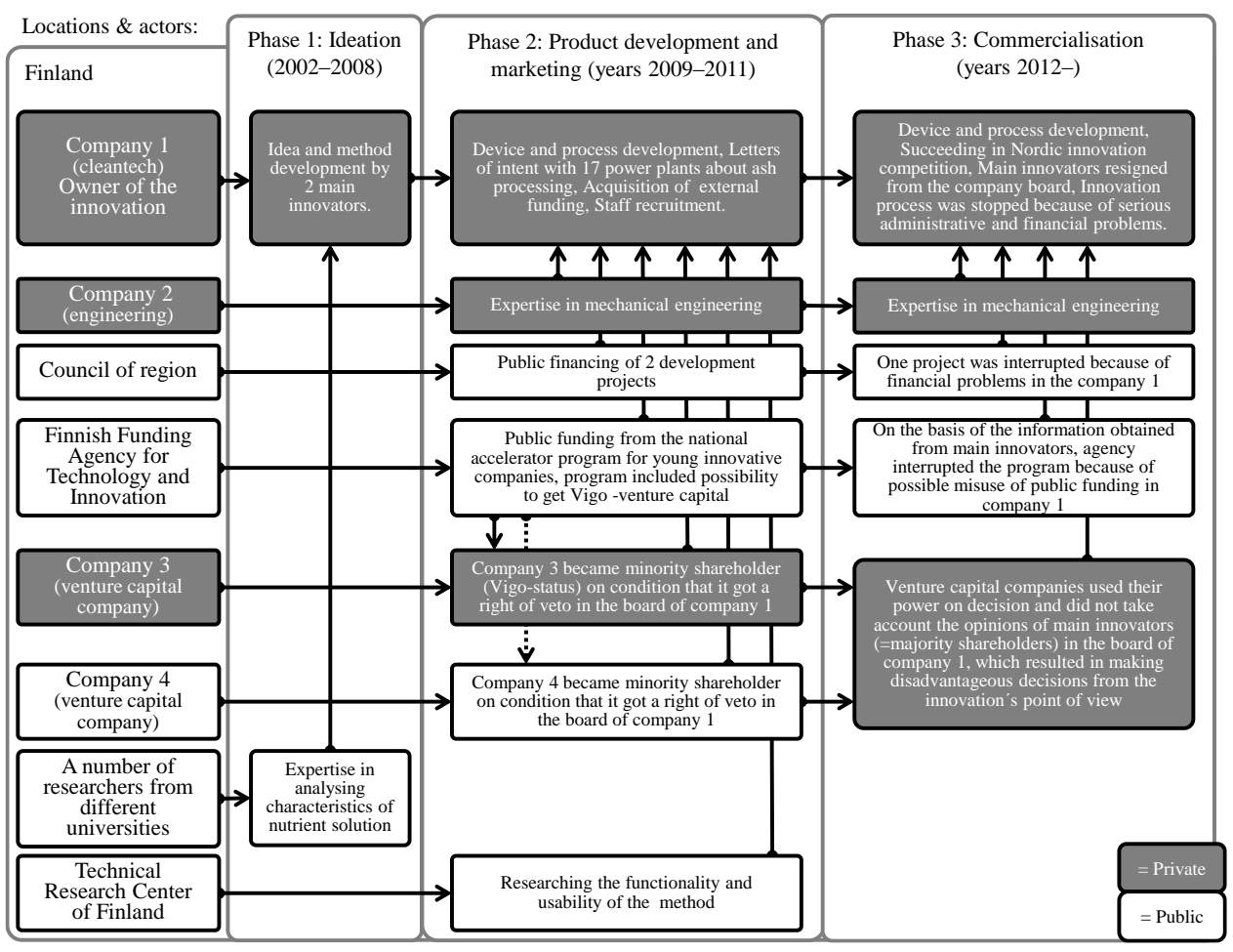

Figure 5. Knowledge biography of innovation case 2

The innovation idea was based on research that was initiated in the 1960s on the utilisation of ash in forest fertilisation by one of the main innovators. In 2002, the first main innovator asked the other main innovator to help him commercialise the nutrient solution, which he had been successful in separating from ash. The other main innovator immediately noticed the business possibilities associated with possessing expertise in ash processing. Subsequently, the main innovators independently began developing the method of ash processing in phase 1 . The objective was to create a method and device that enables the processing and conversion of ash to end-of-waste products. The ash generated by power plants is classified as waste, which causes significant waste handling costs for power plants. 
The most important knowledge was introduced in the development process by the two main innovators, one of whom had significant working experience in the research sector as a professor and manager in a national research centre. The other main innovator had considerable and varied experience in business, development and planning. He also had several degrees from different branches of science and had made 15 patented inventions. External experts mainly participated in the process in phase 2, when the construction of the device was in progress. Public actors participated in the process mainly by financing the development of the method and device. In this case, the influence of the venture capital companies was especially noteworthy. The main innovators inadvertently accepted a shareholders' agreement that gave the venture capital companies a right of veto on the board of company 1 . After a while, the venture capital companies displaced the main innovators from the operational decision-making on the board of company 1 , which caused serious administrative and financial problems and, at the end, the failure of the entire development process, even though the ash processing method was ready and customer acquisition done (letters of intent with 17 power plants).

\subsection{Case 3}

Innovation case 3 is the development of an Internet application to describe electricity price formation and contract rating. Based on the data obtained from the interviews, three innovation phases were identified:

1. Ideation, 2007-2009

2. Product development, 2010-2012

3. Commercialisation, 2013-

Figure 6 illustrates the innovation phases and key knowledge interactions among the actors in case 3. One individual (i.e., main innovator) was the owner of the innovation in phase 1 . Subsequently, the company (i.e., company 1), which was founded in 2010 by the main innovator, was the owner of the innovation. 


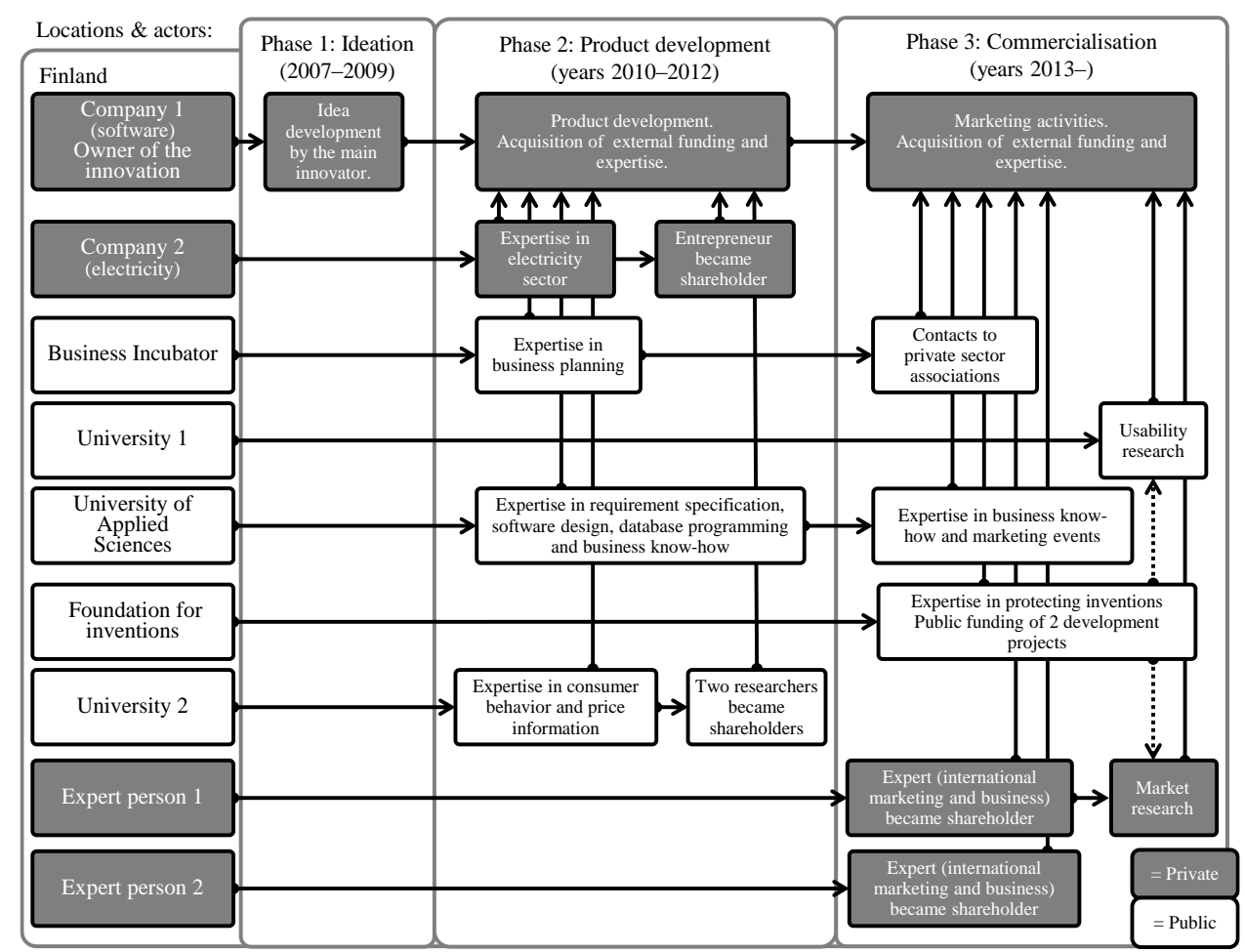

Figure 6. Knowledge biography of innovation case 3

The main innovator got the idea of developing an Internet application to describe electricity price formation and contract rating in 2007 while working in a company that operated as an electricity supplier. At the time, the public believed that traditional internet applications for electricity price comparison only provided a minor benefit to the consumers. This belief of the public gave the innovator the insight that the criteria for price formation in the electricity sector are very non-transparent for customers. The main innovator also noticed that there was no Internet service in the market that transparently presented the criteria for electricity price formation and contract rating. The main innovator independently developed the idea further in phase 1, after which he resigned from his job and founded a new company (i.e., company 1).

The most important knowledge was introduced in the development process by the main innovator, who had extensive and varied working experience in the electricity sector and was a certified electronics technician. In phases 2 and 3, several external experts brought many-sided expertise to the process. New shareholders of company 1 introduced important knowledge to the process in phases 2 and 3. Public actors also played an important role in phases 2 and 3. Public services and the knowledge of universities were effectively utilised in phases 2 and 3.

\subsection{Case 4}




\section{Knowledge Dynamics and Innovation: A Case Study}

Innovation case 4 is the development of a PC-free control system for a forest harvester with remote control possibilities. Based on the data obtained from the interviews, three innovation phases were identified:

1. Ideation, 1996-2002

2. Product development, 2002-2005

3. Commercialisation, 2005-

Figure 7 illustrates the innovation phases and key knowledge interactions among the actors in case 4 . The company owned by the main innovator (i.e., company 1) was the owner of the innovation in phases 1-2. Subsequently, the company (i.e., company 2) which was founded in 2007 was the owner of the innovation.

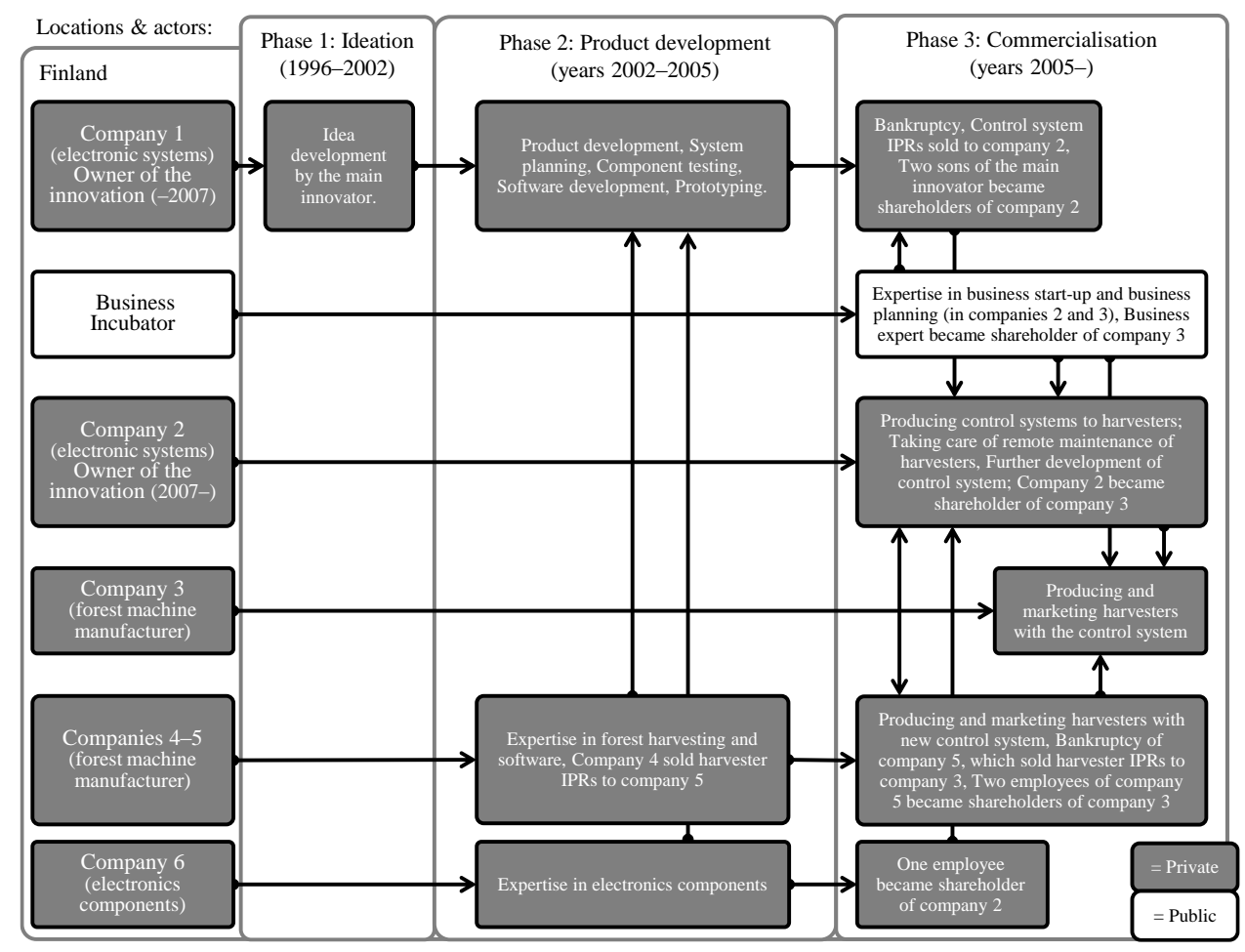

Figure 7. Knowledge biography of innovation case 4

The main innovator got the idea for the control system while he was making electrical installations in forest harvesters. He believed the electronics system that was in place at the time was too complex, too vulnerable to faults and too laborious to make, and he had the idea to replace the numerous hand-adjusted functions with one centralised adjustment and control system.

The most important knowledge was introduced in the development process by the main innovator, who had significant working experience related to planning and engineering different kinds of electricity, electronics and control systems. The main innovator had also studied (self-study) academic publications and manuals related to electrical engineering, electronics and data communication. In the first phase, the main 
innovator further developed his idea independently, in his free time, using his own money.

The knowledge dynamics of the second phase of the innovation process were mainly based on cooperation between three individuals - the main innovator and two external experts. The first expert was an employee of a forest harvester manufacturer and had expertise in programming and software. The second expert was an employee of an electronics component deliverer and had expertise in electronics components.

The knowledge dynamics of the third innovation phase were much more complex than they were in phases 1 and 2. There were more actors involved, and some significant organisational changes took place that affected the development of the new system. This was also the only phase in which a public organisation influenced the innovation process. The prototype of forest harvester with the new control system was presented at a forestry trade fair at the beginning of the third phase. In terms of this step, the harvester manufacturer's knowledge about trade fairs, marketing and the forestry sector was an important factor in marketing the control system as an essential part of the harvester.

\section{Discussion}

This study describes how the innovation process develops from the conception of an idea to a product in four innovation cases and analyses the knowledge dynamics within those processes. The detailed knowledge biographies of the four innovation cases, as presented in the previous chapter, answered research question 1.

Figures 8-11 briefly answer research question 2. In Figures 8-11, the innovation processes occur within a framework of knowledge types and knowledge phases (see Figures 1 and 2), according to the theoretical analysis conducted in this study.

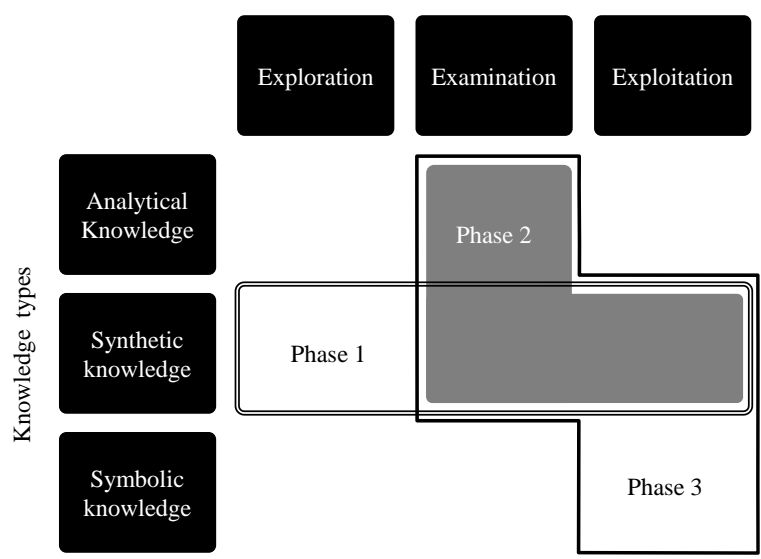

Figure 8. Case 1: The phases of the innovation process, projected through knowledge types and knowledge phases

In case 1, during the first phase of the innovation process (first version), synthetic knowledge was essential, and all the knowledge phases (exploration, examination and exploitation) were identified. The entire planning and manufacturing process was done by the staff, which had extensive experience in tailor-made manufacturing. The first version of the door was manufactured straight to the customer, which meant that no prototypes were made. The second phase (product development) was an examinative and 


\section{Knowledge Dynamics and Innovation: A Case Study}

exploitative knowledge phase in which both analytical and synthetic knowledge were essential. External expertise was necessary when the door improvements were in progress. Two marketing experts were recruited by company 1 in phase 2 , which accelerated the innovation process. The third phase (marketing and internationalisation) was an examinative and exploitative knowledge phase, and all knowledge types were essential. External expertise was especially required in internationalisation activities. One expert was recruited in phase 3 to help in planning and using the new information systems. In phase 3 , the owner company of the innovation learned a lot about customer requirements during the business negotiations with the first big international client company.

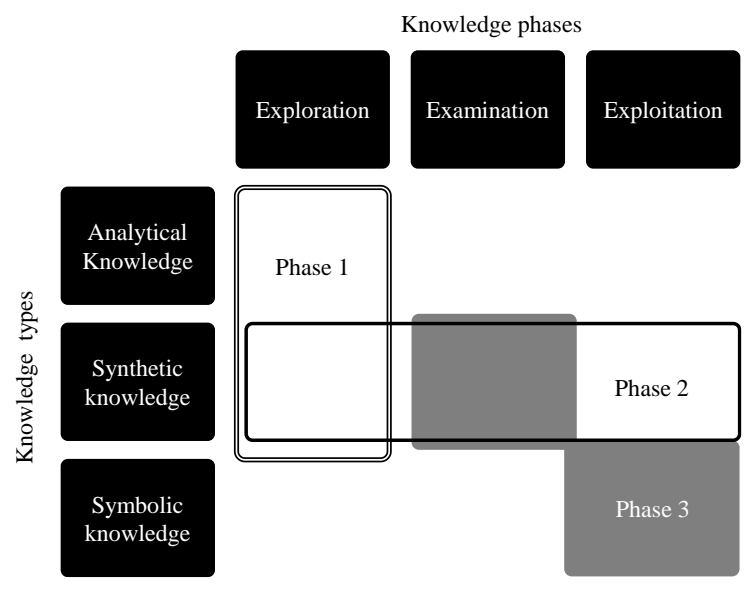

Figure 9. Case 2: The phases of the innovation process, projected through knowledge types and knowledge phases

In case 2, the first phase of the innovation process (ideation) was clearly an explorative knowledge phase in which both analytical and synthetic knowledge were essential. The main innovators were successful in independently developing the idea further because they had many-sided work experience and education. In the second phase (product development and marketing), synthetic knowledge was essential, and all the knowledge phases (exploration, examination and exploitation) were identified. External expertise was required for device development and construction. The business experience of the other main innovator was useful when negotiating letters of intent with power plants and acquisition of external funding in phase 2. Also, several experts were recruited in phase 2 to help to plan marketing and the control system of the processing device. The third phase (commercialisation) was an examinative and exploitative knowledge phase in which synthetic and symbolic knowledge was essential. In phase 3, the peculiar behaviour of the venture capital companies resulted in the failure of the entire development process. The venture capital companies introduced marketing and development knowledge to the process but used their right of veto on the board of the company that owned the innovation in a way that caused the main innovators to resign from the board. 


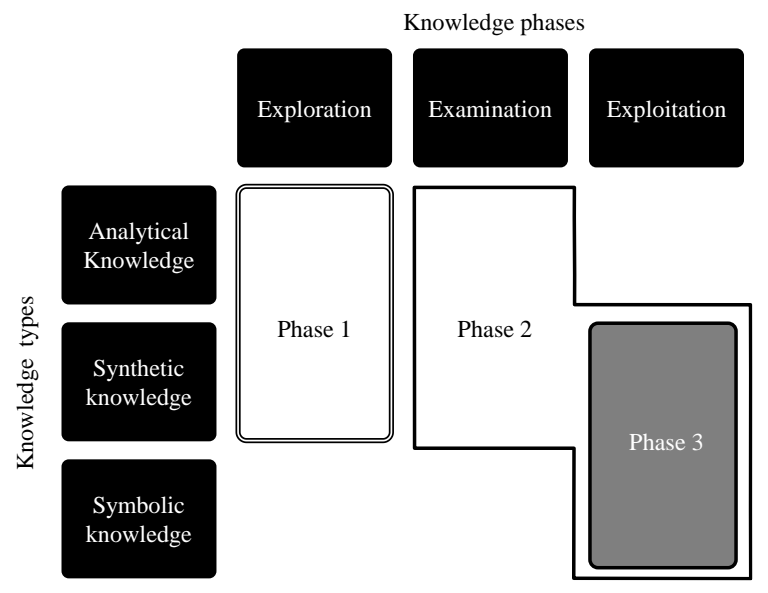

Figure 10. Case 3: The phases of the innovation process, projected through knowledge types and knowledge phases

In case 3, the first phase of the innovation process (ideation) was an explorative knowledge phase in which both analytical and synthetic knowledge were essential. The main innovator was successful in independently developing the idea further, because he had many-sided knowledge about the electricity sector. The second phase (product development) was much more complex; it was an examinative and exploitative knowledge phase wherein all knowledge types were essential. External experts introduced important knowledge to the process, especially for the planning and programming of the application. The new shareholders introduced knowledge about electricity, consumer behaviour and price information to the process in phase 2 . The third phase (commercialisation) represents the exploitative knowledge phase. In this phase, symbolic and synthetic knowledge was decisive. In this phase, the new shareholders introduced important business and marketing knowledge to the process. The foundation for inventions also introduced important knowledge about protecting inventions to the process in phase 3 .

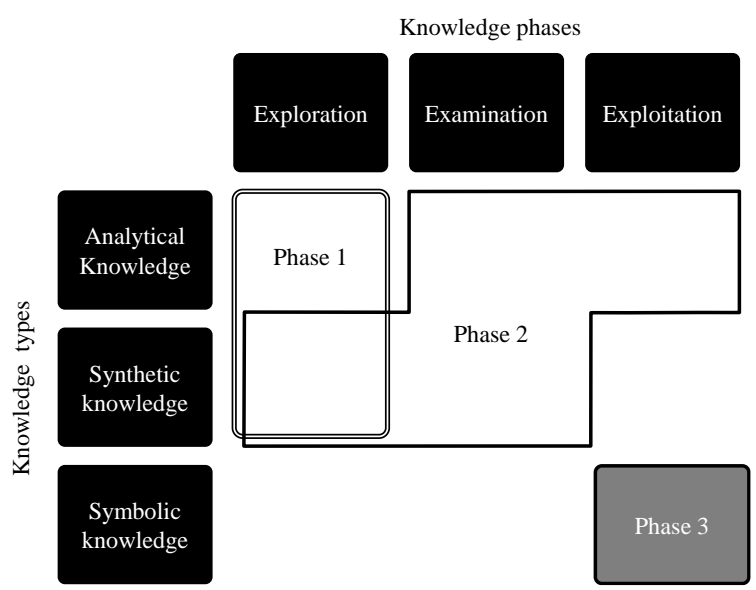

Figure 11. Case 4: The phases of the innovation process, projected through knowledge types and knowledge phases 


\section{Knowledge Dynamics and Innovation: A Case Study}

In case 4, the first phase of the innovation process (ideation) was an explorative knowledge phase in which both analytical and synthetic knowledge were essential. The main innovator was successful in independently developing the idea further, because he had many-sided knowledge about planning and engineering different kinds of electricity, electronics and control systems. The main innovator gained the requisite knowledge for the innovation by undertaking self-study and testing different components and couplings independently. The second phase (product development) was more complex. During this phase, the innovation process was intensive, and all the knowledge phases (exploration, examination and exploitation) were identified. External expertise was required for software development, prototyping and testing the requisite electronics components. The knowledge needed in this phase was analytical and synthetic. The third phase, (commercialisation) represents the exploitative knowledge phase. In this phase, symbolic knowledge was decisive, especially when the prototype was introduced at the trade fair. Symbolic knowledge in this phase played an essential role in planning for and attending the fair and in undertaking other marketing actions, which were undertaken almost entirely on the basis of the actors' previous experience of trade fairs, marketing and the market knowledge of the forestry sector.

From figures 8-11 it can be seen that in all cases, symbolic knowledge exists only in the third innovation phase. The third phase in all the studied innovation processes includes exploitation as the knowledge phase. The symbolic knowledge as the knowledge about representation is understandable in the commercialisation of innovation. In case 1, the first innovation phase differs from other cases, because no analytical knowledge exists in that phase. This is understandable, because the innovation was based on the existing product of the company that owned the innovation; the actors were able to apply existing knowledge. In cases $2-4$, the innovation was an entirely new product, so the process started from the beginning and therefore, analytical knowledge was important. The second innovation phase was clearly the most complex phase in cases $2-4$. In case 1 , the most complex phase was the third phase, which is understandable, because the company that owned the innovation was growing quickly, and the internationalisation process was running during phase 3 .

In cases 2 and 3, the owner of the innovation was a young company, which partially explains the fact that public actors and public funding were more important in the innovation process in these cases. In cases 1 and 4, the owner of the innovation was an older company, which acted independently during the process. In case 1, the owner company acted independently because the innovation was based on the existing product of the company, so already in the baseline, the company had important knowledge from the innovation's point of view. In case 1, the owner company was bigger than owner companies in the other cases, so it had more resources, and thanks to this, better possibilities to act independently. In case 4 , the innovation was an entirely new product, as in cases 2 and 3 . In case 4 , the owner company acted independently because the main innovator had had bad experiences from earlier broad-based development processes and consequently, he wanted to keep the developer group as small as possible in this case. In case 4, the greater part of the important knowledge came from the main innovator, which partially explains why the owner company acted independently in case 4 . In case 1 , other companies provided a large part of the expertise that was required during the process, and numerous public actors were involved. In case 4 , other companies were also important knowledge sources, but only one public actor was involved.

In cases 2 and 3, the companies made several prototypes of the product before marketing activities; however, in case 1, the company manufactured the first version of the door straight to the customer. In case 4, the first version of the control system was presented in a trade fair as an essential part of the forest harvester. 
Despite the differences in the four cases, the basis of the innovations in all the cases was the extensive work experience of the main innovators. This is seen in all cases in phase 1, where the innovation progressed thanks to the knowledge of the main innovators. It is also noteworthy that in all four cases, the companies that owned the innovations collaborated with ease with the actors outside their home region.

The public actors participated most in the innovation process in case 3. Public business services participated in the innovation process in all four cases, but their importance was slighter than it could be estimated on the basis of the amount of public innovation services to which the companies had access. The knowledge of universities was utilised most in case 3, where the main innovator asked the two researchers of the university to become shareholders of the company that owned the innovation.

The knowledge of the subcontractors was most important in case 1, which is understandable, because the innovation was based on the existing product that was produced together with the network of subcontractors in branches that were also useful in the innovation.

Analysing the innovation phases of cases 1-4 in terms of tacit and codified knowledge, it is clear that codified knowledge was needed in every innovation process. The most important codified knowledge in case 1 was the product and component information of subcontractors. Research results of universities and research centres played a significant role in the innovation process of case 2 . The crucial codified knowledge needed in case 3 was the basic data that is located in various databases of different organisations. In case 4, codified knowledge consisted of common manuals, self-study materials, research articles and recognised standards. The studied innovations would not have been processed without the tacit knowledge. The practical skills and the previous experience of the main innovators were the basis of the innovative idea in each case. Personal contacts played a crucial role in ensuring the progression of the innovation process. Also, the reliance between the actors can be seen as tacit knowledge.

In terms of research question 3, knowledge anchoring is viewed in the firm-level context. It was found to play a very interesting role in the innovation processes. The core knowledge needed for the innovations was mainly anchored in the main innovators. Besides that, the important knowledge for the innovation in case 1 was anchored in the personnel of the company that owned the innovation as well as in the personnel of the subcontractors. In case 2, the knowledge was not anchored in the majority of the personnel, because the company was not successful in recruiting employees. However, the knowledge anchoring happened in the partner company. There was a different process of knowledge anchoring in case 3 than in the other cases. The main innovator searched intentionally for the needed knowledge by asking experts to become shareholders in the company. In case 4, the knowledge was anchored in the personnel of the partner companies. The knowledge was also anchored to the new owner company of the innovation through shareholders.

Reviewing the knowledge anchoring by analysing the characteristics of knowledge interactions, it can be said that in all four innovation cases, the number of actors increases, the geographical dimension widens and the cross-sectoral dimension diversifies as the innovation process develops. The multiactor feature means that there are many types of actors, both private and public, in knowledge interactions. In this study, the multiscalar feature is observed by the geographical dimension. The crosssectoral feature describes the variety of branches involved in the innovation. The characteristics of knowledge interactions, as revealed by the findings, are presented in Table 1.

Table 1. Characteristics of knowledge interactions 


\begin{tabular}{|c|c|c|c|c|}
\hline \multicolumn{2}{|c|}{ Knowledge interactions } & $\begin{array}{l}\text { Multiactor feature } \\
\text { (1=narrow, } \\
\text { 2=medium, } \\
\text { 3=large) }\end{array}$ & $\begin{array}{l}\text { Multiscalar feature } \\
\qquad \begin{array}{c}\text { (1=narrow, } \\
\text { 2=medium, } \\
\text { 3=large })\end{array}\end{array}$ & $\begin{array}{c}\text { Cross-sectoral feature } \\
(1=\text { narrow, } \\
2=\text { medium, } \\
3=\text { large })\end{array}$ \\
\hline \multirow{3}{*}{ Case 1} & Phase 1 & 1 & 1 & 1 \\
\hline & Phase 2 & 2 & 2 & 2 \\
\hline & Phase 3 & 3 & 3 & 3 \\
\hline \multirow{3}{*}{ Case 2} & Phase 1 & 2 & 2 & 2 \\
\hline & Phase 2 & 3 & 3 & 3 \\
\hline & Phase 3 & 2 & 3 & 2 \\
\hline \multirow{3}{*}{ Case 3} & Phase 1 & 1 & 1 & 1 \\
\hline & Phase 2 & 3 & 2 & 3 \\
\hline & Phase 3 & 3 & 3 & 3 \\
\hline \multirow{3}{*}{ Case 4} & Phase 1 & 1 & 1 & 1 \\
\hline & Phase 2 & 2 & 2 & 2 \\
\hline & Phase 3 & 3 & 3 & 3 \\
\hline
\end{tabular}

Looking at phase 1 , there is a difference in case 2 compared to the others. The external experts were involved already in phase 1 (multiactor feature). Their impact is seen in other features, too. Surprisingly, the multiactor and cross-sectoral features decreased in phase 3 , which refers to the financial and administrative problems.

A construct validity of this case study is based on a research plan that was used as a road map throughout the study. The case studies used multiple sources of evidence, including interviews and public archive documents, and the chain of evidence was carefully established. Moreover, the key informants reviewed the draft case study reports during the process. The internal validity of the study relies on the research design. The causal relationships were built carefully to avoid any biases. Where external validity is concerned, through analysis of replications in several innovation cases, generalisation from replications to theory is possible (Yin, 1989). For evaluation of the reliability of the study, the following points are highlighted. Case study protocol was used during the data collection phase, the case database was established and the data collection process was documented - the research was conducted so that an auditor could repeat the procedures and arrive at similar kinds of results. Anyhow, the researchers' own paradigm may affect the findings in this type of case study. This affect was minimised by an inclusive and iterative data collection process, which enabled verification of the interpretations during the process. Moreover, several actors were interviewed and several people's views were obtained in the interpretation.

The findings of this study suggest that while developing their services, public actors should pay more attention to catering to the case-specific needs of innovative micro-sized companies. The results of this study show how micro-sized companies can function effectively with limited financial and human resources. On the basis of the information obtained from the four innovation cases analysed in this study, it seems that innovations in micro-sized companies provide a challenge to the regional innovation system, especially in how to reach the innovators at the early stages of the innovation process.

As seen in this study, different kinds of administrative and geographical borders seem to have quite a minor influence on the innovation process from the point of view of the innovation owner. Therefore, it might be useful to discuss whether the focus of public innovation services is wide enough, for example, how prepared the public actors are to cooperate with subcontractors located outside their own region.

In future studies, it would be interesting to use the knowledge biography technique to examine more innovation cases of micro-sized companies in Finland and other countries. We hope to deepen the understanding of knowledge dynamics by emphasising learning 
mechanisms and knowledge management practices that influence the innovations of micro-sized companies.

\section{References}

Anussornnitisarn, P., Sanpanich, S., Phusavat, K. and Kess, P. (2010) 'Sustaining organisational innovation and learning through external knowledge', International Journal of Innovation and Learning, Vol. 7, No. 1, pp.85-99.

Asheim, B.T. and Coenen, L. (2006) 'Contextualising regional innovation systems in a globalising learning economy: on knowledge bases and institutional frameworks', The Journal of Technology Transfer, Vol. 31 No. 1, pp.163-173.

Ayyagari, M., Beck, T. and Demirguc-Kunt, A. (2007) 'Small and medium enterprises across the globe', Small Business Economics, Vol. 29 No. 4, pp.415-434.

Collinge, C., Macneill, S., James, L., Larsson, A., Widmaier, B., Jeannerat, H., Butzin, A. and Vale, M. (2008) Reporting Template WP6 FKD Final Reports, EURODITE: unpublished project internal working paper.

Crevoisier, O. and Jeannerat, H. (2009) 'The territorial knowledge dynamics: from the proximity paradigm to multi-location milieus', European Planning Studies, Vol. 17, No. 8, pp.1223-1241.

Dahlström, M. and Hedin, S. (Eds.) (2010) Regional Trajectories to the Knowledge Economy - Nordic-European Comparisons, Nordic Innovation Centre, Oslo.

Dean, J. (1950) 'Pricing policies for new products', Harvard Business Review, Vol. 28 No. 6, pp.45-53.

Golder, P.N. and Tellis, G.J. (2004) 'Growing, growing, gone: cascades, diffusion, and turning points in the product life cycle', Marketing Science, Vol. 23 No. 2, pp.207218.

Halkier, H., Dahlström, M., James, L., Manniche, J. and Smed Olsen, L. (eds.) (2010) Knowledge Dynamics, Regional Development and Public Policy, Department of History, International and Social Studies, Aalborg University, Alborg.

James, L., Dahlström, M. and Smed Olsen, L. (2010) 'Knowledge Anchoring in European Regions: Policy Implications', in Halkier, H., Dahlström, M., James, L., Manniche, J. and Smed Olsen, L. (Eds.), Knowledge Dynamics, Regional Development and Public Policy, EURODITE, EU.

Kess, P., Phusavat, K. and Takala, J. (2008) 'Managing external knowledge: framework for organisational life cycles', International Journal of Innovation and Learning, Vol. 5, No. 3, pp.255-265.

Markusen, A.R., Hall, P., and Glasmeier, A. (1986) High Tech America: The What, How, Where, and Why of the Sunrise Industries, Allen \& Unwin, Boston.

Phusavat, K., Sanpanich, S., Kess, P. and Muhos, M. (2009) 'The roles of external knowledge in organisational learning and development', International Journal of Innovation and Learning, Vol. 6, No. 5, pp.537-549.

Schutjens, V. and Stam, E. (2003) 'The evolution and nature of young firm networks: a longitudinal perspective', Small Business Economics, Vol. 21, No. 2, pp.115-134.

Storey, D.J. (2003) 'Entrepreneurship, small and medium sized enterprises and public policy', in Acs, Z.J. and Audretsch, D.B. (Eds.): Handbook of Entrepreneurship Research, pp.473-511, Dordrecht.

Strambach, S. (2008) 'Knowledge-intensive business services (KIBS) as drivers of multilevel knowledge dynamics', International Journal of Services Technology and Management, Vol. 10, No. 2, pp.152-174. 
19 Knowledge Dynamics and Innovation: A Case Study

Yin, R.K. (1989) Case Study Research: Design and Methods, Sage Publications, Beverly Hills. 